\title{
Completely Randomised Design (CRD) Analysis - by Manual and MS-Excel
}

\author{
V. Sekhar ${ }^{1 *}$, K. Umakrishna ${ }^{1}$, V. Srinivasa Rao $^{2}$ and Ch. Satyavani ${ }^{3}$ \\ ${ }^{1}$ Department of Statistics, ${ }^{3}$ College of Horticulture, Venkataramannagudem, Dr. YSR \\ Horticultural University, West Godavari (District), \\ Andhra Pradesh (State), India, PIN-534101 \\ ${ }^{2}$ Department of Statistics, Agricultural College, Bapatla, Acharya NG Ranga Agricultural \\ University, Guntur (District), Andhra Pradesh (State), India, PIN-522101
}

*Corresponding author

\section{A B S T R A C T}

\section{Keywords CRD, ANOVA, CD \\ Article Info \\ Accepted: \\ 10 February 2019 \\ Available Online: \\ 10 March 2019}

This study was attempted to find out best yield variety among 10 Gerebera varieties by using Completely Randomised Design. Terra Juba found best yield variety based on critical difference. CRD is most suitable for laboratory experiments, pot experiments and green house experiments.

\section{Introduction}

Completely randomised design is the one in which all the experimental units are taken in a single group which are homogeneous as far as possible. For example, all the field plots constituting the group are having the same soil fertility, soil depth, soil texture, soil moisture, etc.; This design is also called as non-restriction design $(1,2)$.

CRD follows two basic principles of experimental Designs

1. Replication

2. Randomisation
CRD follows ANOVA-Iway classification

\section{Advantages of CRD}

This design has complete flexibility, i.e., any number of treatments and replicates for each treatment can be taken,

Whole experimental material can be utilised in CRD.

CRD has maximum degrees of freedom for experimental error.

CRD layout is very easy and analysis of data is simplest as compared to any other design.

Missing observation creates no problem in analysis of data. The analysis is carried out in 
the usual manner neglecting the missing plot as if it was not there in the experiment.

\section{Disadvantages of CRD}

Local control is absent in CRD

It is applicable for homogeneous experimental units and not for heterogeneous experimental area

\section{Materials and Methods}

In a homogeneous experimental area, a varietal trial on gerbera was conducted with 10 varieties each replicated 3 times at College of Horticulture, Venkataramannagudem in the year 2016. Flower yield in grams per plant are furnished here under analyse the data and draw your conclusion $(3,4,5)$

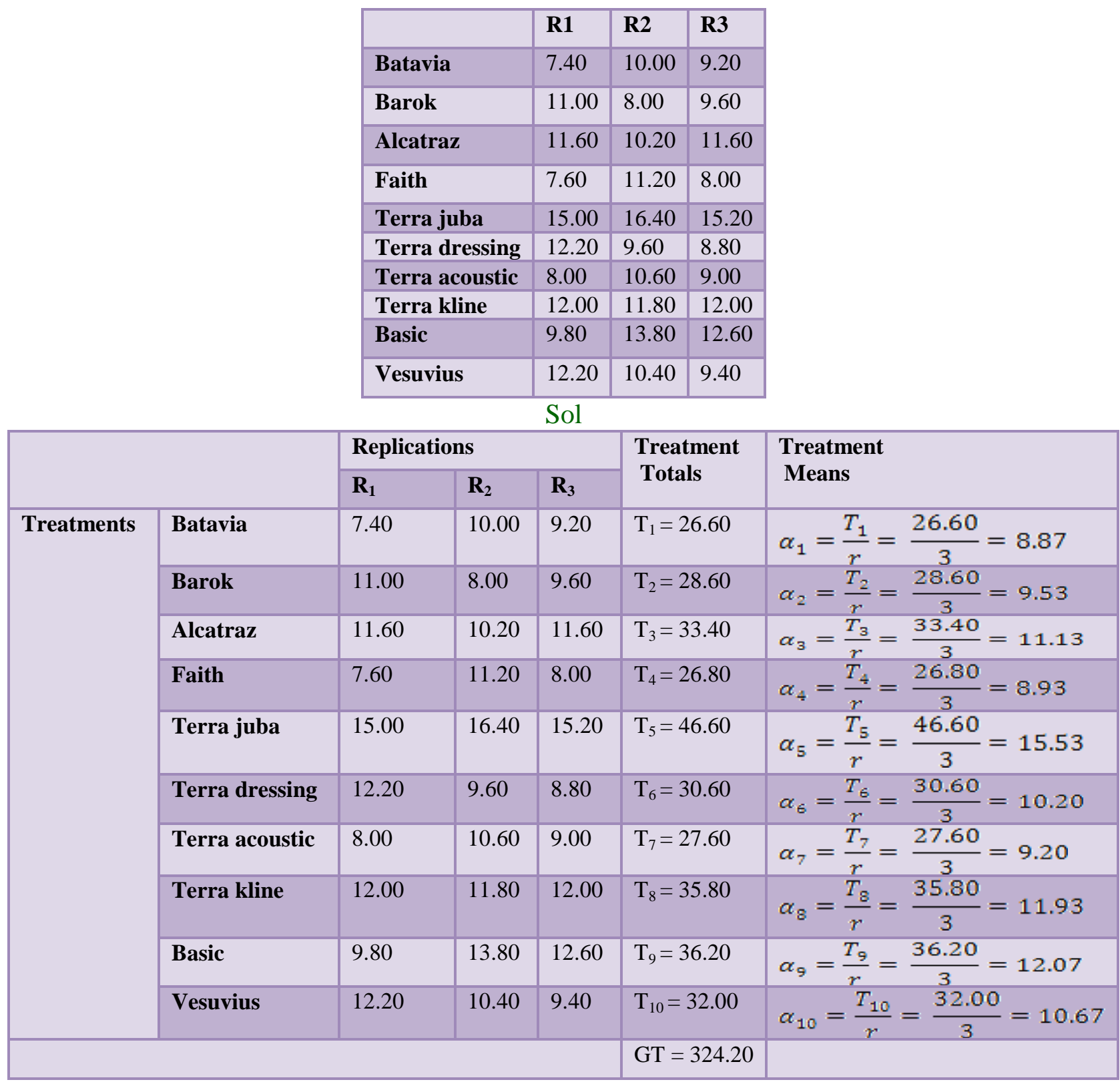


The mathematical model for CRD is $x_{i j}=\mu+\alpha_{i}+E_{i j}$

$H_{0}$ :There is no significant difference among mean of varieties

$H_{0}: \alpha_{1}=\alpha_{2}=\ldots \ldots \ldots \ldots=\alpha_{10}$

$H_{1}$ :There is significant difference among mean of varieties

$H_{0}: \alpha_{1} \neq \alpha_{2} \neq \ldots \ldots \ldots \ldots \alpha_{10}$

$\mathrm{k}$ is no. Of varieties $=10$

$\mathrm{r}$ is no. Of replications $=3$

$G M=\frac{G T}{k r}=\frac{324.20}{10 * 3}=\frac{324.20}{30}=10.81$

$C F=\frac{G T^{2}}{k r}=\frac{324.20^{2}}{10 * 3}=\frac{324.20 * 324.20}{30}=\frac{105105.64}{30}=3503.521$

$$
\begin{aligned}
& \text { Total SS }=\sum_{i=1}^{k} \sum_{j=1}^{r} x_{i j}^{2}-C F=\left(x_{1,1}^{2}+x_{1,2}^{2}+\ldots \ldots+x_{10,3}^{2}\right)-C F \\
& =\left(7.4^{2}+10^{2}+\ldots \ldots \ldots \ldots+9.4^{2}\right)-3503.521 \\
& =3655.56-3503.521=152.04
\end{aligned}
$$$$
\operatorname{TrtSS}=\frac{T_{1}^{2}+T_{2}^{2}+\cdots \cdots \cdots+T_{k}^{2}}{r}-C F
$$$$
=\frac{26.6^{2}+28.6^{2}+\cdots \ldots \ldots+32^{2}}{3}-3503.521
$$$$
=\frac{10845.08}{3}-3503.521
$$$$
=3615.03-3503.521=111.51
$$

Error SS $=$ Total SS - TrtSS

\begin{tabular}{|c|c|c|c|c|c|}
\hline $\begin{array}{l}\text { Sources } \\
\text { Of } \\
\text { Variation }\end{array}$ & $\begin{array}{l}\text { Degrees } \\
\text { Of } \\
\text { freedom }\end{array}$ & $\begin{array}{l}\text { Sum } \\
\text { of } \\
\text { Squares }\end{array}$ & $\begin{array}{l}\text { Mean } \\
\text { Sum of } \\
\text { Squares }\end{array}$ & F-Cal & F-tab \\
\hline Treatments & $\begin{array}{l}\mathrm{k}-1 \\
10-1=9\end{array}$ & $\begin{array}{l}\text { Trt SS = } \\
111.51\end{array}$ & $\begin{array}{l}\text { Trt MSS }=\frac{\operatorname{Trt} . S S}{k-1} \\
=\frac{111.51}{9}=12.39\end{array}$ & $\begin{array}{l}\frac{\text { Trt.MSS }}{\text { Error MSS }} \\
\frac{12.39}{2.03}=6.10\end{array}$ & $\begin{array}{l}F_{k-1, k}(r-1) \\
F_{9,20}=2.39\end{array}$ \\
\hline Error & $\begin{array}{l}\mathrm{k}(\mathrm{r}-1) \\
10(3-1)=20\end{array}$ & $\begin{array}{l}\text { Error } \mathrm{SS}= \\
40.53\end{array}$ & $\begin{array}{l}\text { Error MSS }=\frac{\text { Error. } S}{k(r-1)} \\
=\frac{40.53}{20}=2.03\end{array}$ & --- & --- \\
\hline Total & $\begin{array}{l}\mathrm{kr}-1 \\
10 * 3-1=29\end{array}$ & $\begin{array}{l}\text { Total } \mathrm{SS}= \\
152.04\end{array}$ & --- & --- & --- \\
\hline
\end{tabular}

$$
=152.04-111.51=40.53
$$

Prepare the following ANOVA Table 


\section{Analysis procedure of CRD in MS-Excel}

In MS-Excel sheet, enter dataas below picture

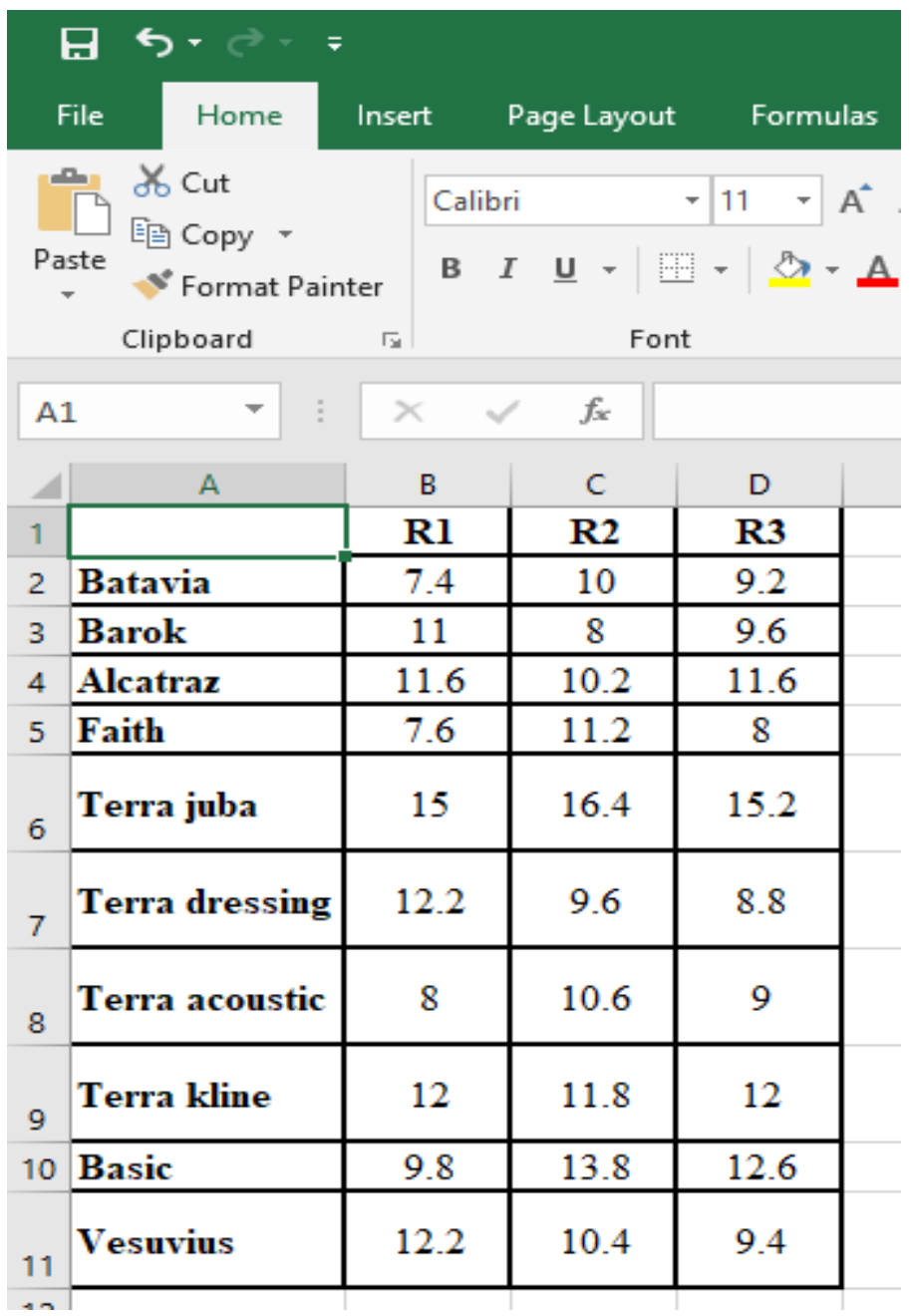

Select Data menu in Excel $\rightarrow$ choose Data analysis

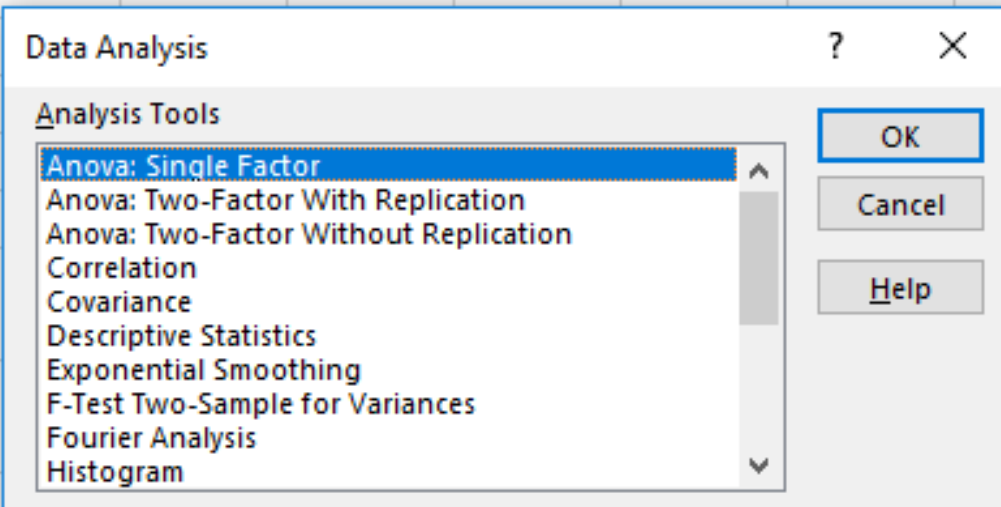




\section{Choose Anova: Single Factor $\rightarrow$ press OK}

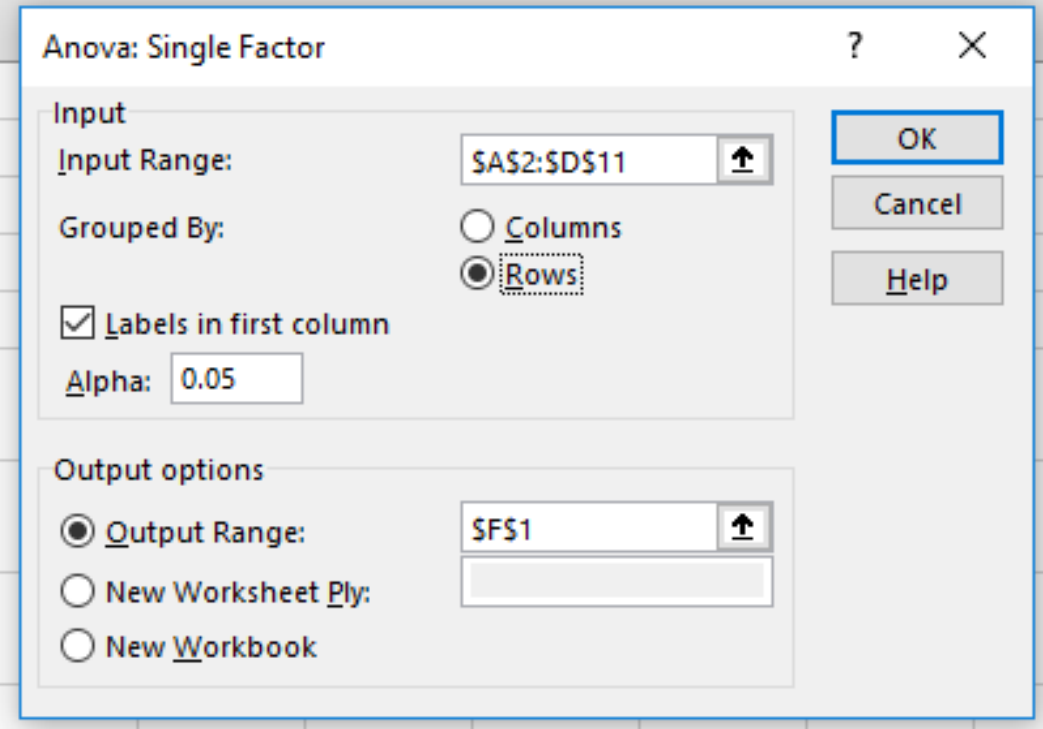

Give Input Range: $\$ \mathrm{~A} \$ 2: \$ \mathrm{D} \$ 11 \rightarrow$ choose Rows option under Grouped By: $\rightarrow$ Put tick mark on Labels in first Column $\rightarrow$ Give Alpha: 0.05 for $5 \%$ level of significance $\rightarrow$ Give output Range\$F\$1 under Output options (i.e. Output will display from F1 cell) $\rightarrow$ press OK

\begin{tabular}{|c|c|c|c|c|c|c|}
\hline $\mathrm{F}$ & G & $\mathrm{H}$ & 1 & J & K & L \\
\hline \multicolumn{7}{|l|}{ Anova: Single Factor } \\
\hline \multirow{2}{*}{\multicolumn{7}{|c|}{ SUMMARY }} \\
\hline & & & & & & \\
\hline Groups & Count & Sum & Average & Variance & & \\
\hline Batavia & 3 & 26.6 & 8.866667 & 1.773333 & & \\
\hline Barok & 3 & 28.6 & 9.533333 & 2.253333 & & \\
\hline Alcatraz & 3 & 33.4 & 11.13333 & 0.653333 & & \\
\hline Faith & 3 & 26.8 & 8.933333 & 3.893333 & & \\
\hline Terra juba & 3 & 46.6 & 15.53333 & 0.573333 & & \\
\hline Terra dressing & 3 & 30.6 & 10.2 & 3.16 & & \\
\hline Terra acoustic & 3 & 27.6 & 9.2 & 1.72 & & \\
\hline Terra kline & 3 & 35.8 & 11.93333 & 0.013333 & & \\
\hline Basic & 3 & 36.2 & 12.06667 & 4.213333 & & \\
\hline Vesuvius & 3 & 32 & 10.66667 & 2.013333 & & \\
\hline & & & & & & \\
\hline \multicolumn{7}{|l|}{ ANOVA } \\
\hline Source of Variation & SS & $d f$ & MS & $F$ & $P$-value & Fcrit \\
\hline Between Groups & 111.5053 & 9 & 12.38948 & 6.113231 & 0.000377 & 2.392814 \\
\hline Within Groups & 40.53333 & 20 & 2.026667 & & & \\
\hline Total & 152.0387 & 29 & & & & \\
\hline
\end{tabular}

In the ANOVA table of above picture, Between Groups means Treatments or Varieties and Within Groups means Error 


\section{Results and Discussion}

Here, F-cal(6.10) value is > F-tab at 5\% level of significance with $F_{k-1, k(r-1)} F_{9,20}=2.39$ and also greater than F-tab at $1 \%$ level of significance with $F_{k-1, k(r-1)} F_{9,20}=3.46$.

So, we reject Null Hypothesis. i.e., There is highly significant difference among variety means.

\section{Standard Errors}

$S E M=\sqrt{\frac{\text { Error MSS }}{r}}=\sqrt{\frac{2.03}{3}}=0.82$

$S E D=\sqrt{2} * S E M=\sqrt{2} * 0.82=1.41 * 0.82=1.16$

$C D=S E D * t_{\text {tab at error d.f }}=1.16 * 2.09=2.42$

\section{Bar notation}

We arrange the treatment means into decreasing order. Now compare treatment means difference with $\mathrm{CD}$ value. If the difference between treatment means is less than $\mathrm{CD}(2.42)$, then underline. Otherwise leave it.

- $\quad$ Compare mean differences of Terra Juba and Basic with CD $15.53-12.07=3.46$ i.e., 3.46 is greater than $\mathrm{CD}(2.42)$. So, don't underline between these treatments

- Compare mean differences of Basic and Terra klinewith CD $12.07-11.93=0.14$ i.e. 0.14 is less than $\mathrm{CD}(2.42)$. So, underline between these treatments

\begin{tabular}{|l|l|l|l|l|l|l|l|l|l|}
\hline $\begin{array}{l}\text { Terra } \\
\text { juba }\end{array}$ & Basic & $\begin{array}{l}\text { Terra } \\
\text { kline }\end{array}$ & Alcatraz & Vesuvius & $\begin{array}{l}\text { Terra } \\
\text { dressing }\end{array}$ & Barok & $\begin{array}{l}\text { Terra } \\
\text { acoustic }\end{array}$ & Faith & Batavia \\
\hline 15.53 & 12.07 & 11.93 & 11.13 & 10.67 & 10.20 & 9.53 & 9.20 & 8.93 & 8.87 \\
\hline
\end{tabular}

Those pairs underscored are Non-significant Those pairs not scored are Significant

In conclusion, among 10 varieties $5^{\text {th }}$ variety (Terra Juba) is found best variety.

\section{References}

1. A text Book of Agricultural Statistics- R. Rangaswamy, New Age International Publishing Limited, Hyderabad, 2010.

2. Statistics for Agricultural Sciences- G. Nageswara Rao, BS Publications, Hyderabad (Second Edition), 2007.
3. Programmed Statistics (QuestionsAnswers) $2^{\text {nd }}$ Edition- B. L. Agarwal, New Age International Publishing Limited, New Delhi, 2009.

4. Practical manual of STCA-101 (for class use only)- Dr. V. Srinivasa Rao and R. Srinivasulu, Agricultural College, Bapatla, 2013.

5. MS-Excel: Statistical Procedures- Cini Varghese, IASRI, Library Avenue, New Delhi-110012.

\section{How to cite this article:}

Sekhar, V., K. Umakrishna, V. Srinivasa Rao and Satyavani, Ch. 2019. Completely Randomised Design (CRD) Analysis - by Manual and MS-Excel. Int.J.Curr.Microbiol.App.Sci. 8(03): 954-959. doi: https://doi.org/10.20546/ijcmas.2019.803.114 